
\title{
The Role of Polyunsaturated Fatty Acids in Infant
Nutrition
}

Robert A. Gibson

Director, Infant Nutrition Research Centre, Flinders Medical Centre, Adelaide, SA 5042, Australia.

The need for long chain polyunsaturated fatty acids (LCPUFA), such as docosahexaenoic acid (DHA, 22:6n-3) and arachidonic acid (AA, 20:4n-6), in the diet of infants to achieve full developmental potential is a matter of intense investigation by several research groups world-wide. It has been widely reported that breast fed infants are able to perform better on tests that assess neurodevelopmental outcomes than formula fed infants. Although breast milk contains LCPUFA that are absent from formula, it is necessary to demonstrate that any beneficial effects of breast milk on infant development are purely attributed to the presence of. LCPUFA in breast milk and their absence from formula to establish causality. The hypothesis that dietary DHA is associated with developmental outcome needs to be plausible, the effect must be consistent, specific and independent of confounding factors. The hypothesis is certainly plausible. DHA is avidly incorporated and retained in brain cerebral phospholipids and a most consistent finding has been the lower level of cerebral DHA in the brains of formula fed infants (receiving no DHA) relative to those fed breast milk (receiving DHA). The formula fed infants in these studies were generally fed formulas with adequate ALA levels and this may indicate a nutritional requirement for preformed DHA. 
Studies involving preterm infants have shown a clear requirement for DHA for full visual and neural development. These results are consistent with primate studies. However, intervention studies with term infants that have attempted to improve the DHA supply of infant formula and hence infant development have not yielded consistent results. Some randomised studies have demonstrated improved visual and developmental indices in supplemented over unsupplemented infants, others have failed to demonstrate an effect. This disparity could be due to methodological and environmental differences. It is also notable that supplemental regimens have not specifically added DHA and have included other LCPUFA raising the question as to the specificity of the effect. However, only tissue DHA levels have consistently correlated with outcomes. Collectively these studies suggest a positive of LCPUFA for all infants with little evidence of harm. The need for larger, long term studies controlling for effect modifiers is essential to clarify the issues. 